\title{
Ultrastructural Remodeling of the Neurovascular Unit in the Female Diabetic db/db Model-Part III: Oligodendrocyte and Myelin
}

\author{
Melvin R. Hayden 1,2,*, Deana G. Grant ${ }^{3}$, Aranyra Aroor ${ }^{1,2,4} \mathbb{D}$ and Vincent G. DeMarco ${ }^{1,2,4,5}$ \\ 1 Diabetes and Cardiovascular Center, University of Missouri School of Medicine, Columbia, MO 65212, USA; \\ aroora@health.missouri.edu (A.A.); demarcov@missouri.edu (V.G.D.) \\ 2 Division of Endocrinology and Metabolism, Department of Medicine, University of Missouri, \\ Columbia, MO 63212, USA \\ 3 Electron Microscopy Core Facility, University of Missouri, Columbia, MO 65211, USA; \\ GrantDe@missouri.edu \\ 4 Research Service, Harry S. Truman Memorial Veterans Hospital, Columbia, MO 65212, USA \\ 5 Department of Medical Pharmacology and Physiology, University of Missouri, Columbia, MO 65212, USA \\ * Correspondence: mrh29pete@gmail.com; Tel.: +1-573-346-3019
}

Received: 9 October 2018; Accepted: 5 November 2018; Published: 8 November 2018

\begin{abstract}
Obesity, insulin resistance, and type 2 diabetes mellitus are associated with diabetic cognopathy. In this study, we tested the hypothesis that neurovascular unit(s) (NVU), oligodendrocytes, and myelin within cerebral cortical grey matter and deeper transitional zone regions between the cortical grey matter and white matter may be abnormal. The monogenic $\left(L e p r^{d b}\right)$ female diabetic $\mathrm{db} / \mathrm{db}$ [BKS.CgDock $\left.7^{m}+/+L e p r^{d b} / \mathrm{J}\right]$ (DBC) mouse model was utilized for this ultrastructural study. Upon sacrifice (20 weeks of age), left-brain hemispheres of the DBC and age-matched non-diabetic wild type control C57BL/KsJ (CKC) mice were immediately immersion-fixed. We found prominent remodeling of oligodendrocytes with increased nuclear chromatin condensation and volume and increased numbers of active myelination sites of the cytoplasm in transition zones. Marked dysmyelination with outer myelin lamellae sheath splitting, separation, and ballooning with aberrant mitochondria in grey matter and similar myelin remodeling changes with marked disarray with additional axonal collapse in transitional zones in DBC as compared to CKC models.
\end{abstract}

Keywords: db/db mouse model; myelin; neurovascular unit; oligodendrocyte; subcortical white matter; type 2 diabetes

\section{Introduction}

Myelin is a multilayered lamellar sheath that enwraps axons in the grey and white matter in the brain and is synthesized in the brain by the oligodendroglia. The myelin sheath provides axonal protection and allows the saltatory conduction of the action potential. Oligodendrocytes (OLs), oligodendrocyte precursor cells (OPC), and oligodendrocyte lineage cells are specialized glial cells responsible for the synthesis, wrapping ensheathment, and compacting of myelin in myelinated axons [1-3]. Rudolf Ludwig Virchow (1821-1902) introduced the term myelin and defined it as medullary matter (Markstoff), or myeline, that in extremely large quantity fills up the interval between the axon axis-cylinder and the sheath in primitive nerve-fibers [4].

Michalski and Kothary [1] have set forth a paradigm for development of mature OLs in four distinct phases: (i) the birth, migration and proliferation of OPCs, that occurs in waves; followed by (ii) morphological differentiation with the OL establishing an expansive synthetic 
network of OL cytoplasmic processes; followed by (iii) axonal contact, which leads to myelin wrapping - ensheathment of targeted neuronal axons with ensuing generation of extremely electron dense and compact myelin with an enduring (iv) long-term trophic and metabolic support system for maintenance and protection of myelinated neurons [1].

As OPCs differentiate into mature OLs they undergo an extensive development of active cytoplasmic reorganization of cytoskeleton proteins and endoplasmic reticulum, which increases their intermediate cytoplasmic electron density and helps to identify them when studying with transmission electron microscopy (TEM) (Figure 1). Identifying characteristics have been presented in Part I Table 1 [5] as follows: Oligodendrocytes are intermediate in size and their thinned cytoplasm also have an intermediate electron density that is helpful when comparing to astrocytes and microglia. They also may occur in groups or nests and are more often found in the white matter regions of the brain. In addition to their small cytoplasmic volume, they have multiple protoplasmic extensions, which allow OLs to concurrently myelinate multiple axons [1-4].

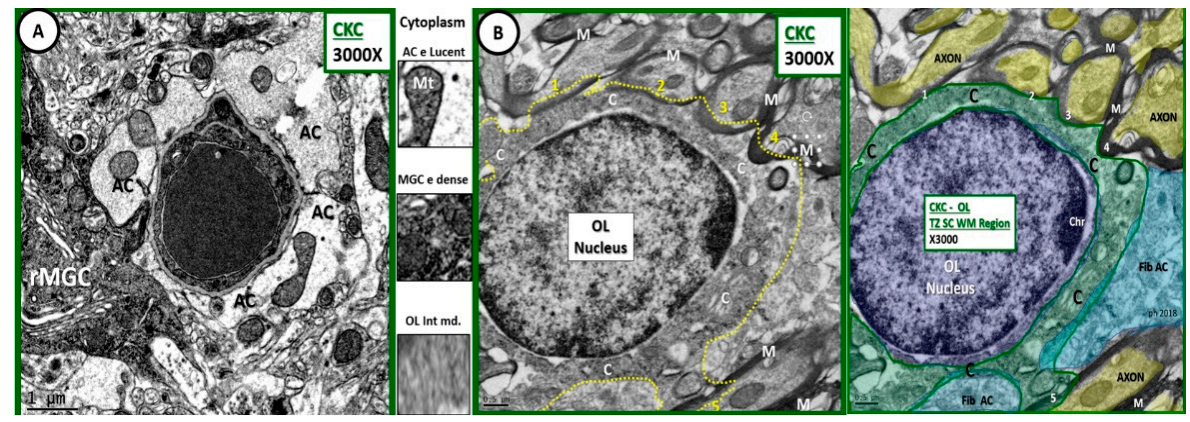

Figure 1. Comparison of astrocyte, ramified microglial cell, and oligodendrocyte to illustrate intermediate electron dense and thin cytoplasm. (A) Depicts an astrocyte (AC) with electron lucent cytoplasm and a ramified microglial cell (rMGC) with its electron dense cytoplasm. (B) Illustrates a thinned intermediate electron dense cytoplasm of the oligodendrocyte (OL) obtained from the transition zone in subcortical white matter (TZ SCWM) just deep to layer VI as compared to ACs and microglia cells. Note that the OL is in the process of enwrapping at least 5 axons (1-5) with myelin in (B) and its pseudo-colored image, far right. Between $(\mathbf{A}, \mathbf{B})$ the cytoplasm has been cut from each cell to further demonstrate the intermediate electron (e) density of the OL cytoplasm. Also note the small amount of cytoplasm as compared to nucleus volume as well as the incorporation of myelin at its outer margins of the cytoplasm plasmalemma. A pseudo-colorized image is inserted far right to aid in identifying OL nucleus (purple); cytoplasm (C green); fibrous astrocyte (fib AC blue); axons (yellow). Magnification $\times 3000$; scale bar $=1 \mu \mathrm{m}$ in A and $0.5 \mu \mathrm{m}$ in (B). C: cytoplasm; MGC: microglial cell; M: myelin; CKC: age-matched non-diabetic wild type control C57BL/KsJ.

The small cytoplasmic volume of oligodendrocyte may be related to its function of constantly supplying its plasma membrane (up to three-times its volume of plasma membranes) in order to create the multiple myelin lamellar sheaths. These myelin lamella that enwrap multiple unmyelinated axons increase the speed of neuronal transmission of their action potentials via membrane depolarization by high-density voltage-gated sodium channels that creates the saltatory (jumping) conduction of their action potentials and signaling at the nodes of Ranvier (Figure 1B). Importantly, this saltatory conduction allows electrical nerve signals to be propagated long distances at high rates without any degradation of the action potential signaling [1-4].

Myelin also serves as a protective sheath of myelinated neurons in order to provide for long-term axonal integrity and maintenance survival. Myelinated axons are present in the cortical grey matter; however, they are the most abundant within the white matter and give rise to the white matter tracts. The bulk of myelinated axons localizes within the white matter, which are important for carrying large amounts of information from one region of the brain to distant regions and must rapidly transmit this 
information. This rapid transmission is primarily due to its compacted electron dense myelin sheaths. Therefore, if myelin undergoes any significant abnormal remodeling change there may be a delay in the in the arrival of information to the more distant regional neurons with subsequent cognitive impairment [6,7].

\section{Materials and Methods}

\subsection{Animal Models}

As previously presented in Part I [5]: All animal studies were approved by the Institutional Animal Care and Use Committees at the Harry S Truman Memorial Veterans' Hospital and University of Missouri, Columbia, MO, USA (No. 190), and conformed to the Guide for the Care and Use of Laboratory Animals published by the National Institutes of Health (NIH). Eight-week-old female $\mathrm{db} / \mathrm{db}$ [BKS.Cg-Dock7 $7^{m}+/+$ Lepr $\left.^{d b} / \mathrm{J}\right]$ (DBC) and wild-type control C57BLKS/J (CKC) mice were purchased from the Jackson Laboratory (Ann Harbor, MI, USA) and were housed under standard laboratory conditions where room temperature was $21-22{ }^{\circ} \mathrm{C}$, and light and dark cycles were $12 \mathrm{~h}$ each. Two cohorts of mice were used: lean non-diabetic controls (CKC, $n=3)$, and obese, insulin-resistant, diabetic $\mathrm{db} / \mathrm{db}(\mathrm{DBC}, n=3)$, which were sacrificed for study at 20 weeks of age.

\subsection{Tissue Collection and Preparation}

Have been previously presented in Part I [5].

\section{Results}

\subsection{Dysmyelination in the Cortical Grey Matter Primarily Layer III}

Splitting and separation of myelin lamellar sheaths (dysmyelination) in the cortical grey matter layer III was readily observed in the female diabetic $\mathrm{db} / \mathrm{db}\left[\mathrm{BKS}\right.$.CgDock $\left.7^{m}+/+L e p r^{d b} / \mathrm{J}\right](\mathrm{DBC})$ as compared to the nondiabetic age-matched non-diabetic wild type control C57BL/KsJ (CKC) models. Additionally, axonal remodeling was noted, which consisted of aberrant mitochondria and increased axonal cytoplasm electron density as compared to the nondiabetic CKC model (Figures 2 and 3).

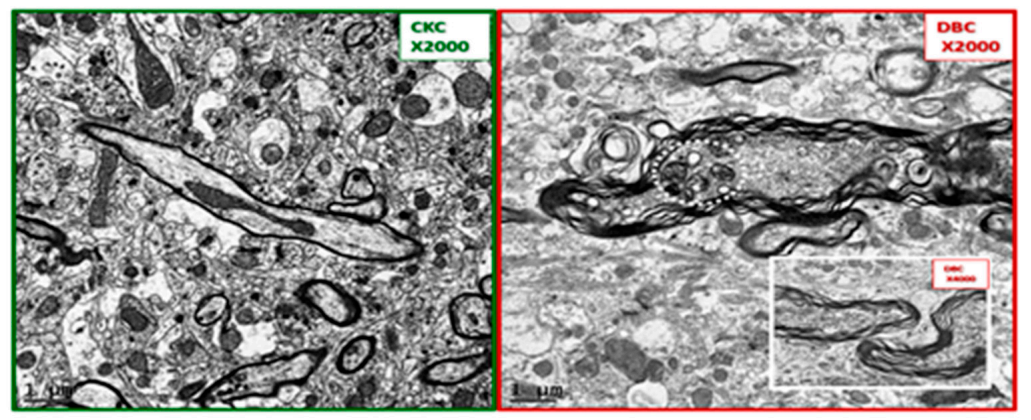

Figure 2. Comparison of control CKC and diabetic diabetic db/db [BKS.CgDock $\left.7^{m}+/+L e p r d b / J\right](D B C)$ myelination in layer III cortical grey matter. These panels depict the marked difference between the myelination of axons in the cortical grey matter of Layer III. Note in the diabetic DBC panel (to the right) the marked splitting and separation of myelin (dysmyelination) and the grouping of suspected aberrant mitochondria (encircled white dashed line). The insert in the DBC panel is from another model in cortical layer III with dysmyelination. Also note the increased thickness of myelin which measures approximately $0.5 \mu \mathrm{m}$ in the DBC as compared to the $0.1 \mu \mathrm{m}$ thickness of the myelin in the CKC model. Also note the elongated axonal mitochondria in the CKC model in contrast to the smaller rounded mitochondria in the DBC. Importantly, note the increase in electron density of the axoplasm of the neuron in the DBC as compared to the CKC. Magnification $\times 2000 ;$ bar $=1 \mu \mathrm{m}$. Magnification of insert $\times 4000$ and scale bar $=0.5 \mu \mathrm{m}$. 


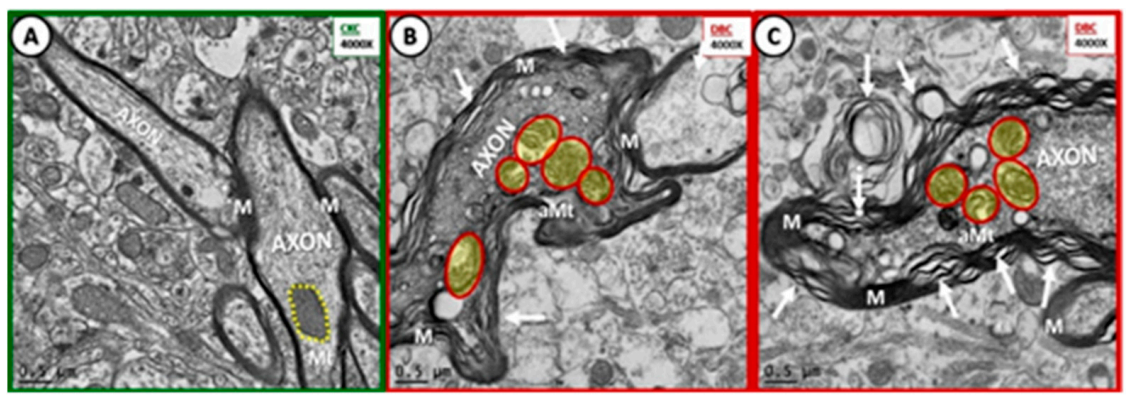

Figure 3. Dysmyelination and aberrant mitochondria in cortical grey matter layer III of diabetic DBC models. (A) Depicts the normal compact myelin (M) ensheathing the axons of the nondiabetic control CKC models and note the normal electron dense mitochondria (Mt) (encircled by yellow dashed line). Panels (B,C) depict the marked splitting and separation of myelin ensheathment in DBC models (arrows). Also note the aberrant $\mathrm{Mt}(\mathrm{aMt}$ - - (pseudo-colored yellow with red lines) and increased electron dense cytoplasm in the DBC. Magnification $\times 4000$; bar $=0.5 \mu \mathrm{m}$.

Also, ballooning of myelinated axons was detected, but not with the frequency of myelin splitting and separation (Figure 4).
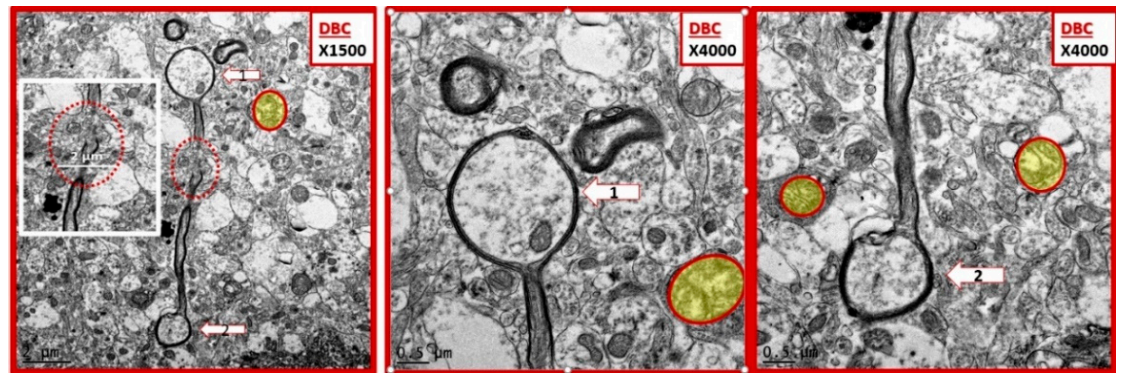

Figure 4. Examples of ballooning in myelinated axons of diabetic DBC models in cortical grey matter. This three-panel image depicts ballooning of myelinated axons that were only observed in the diabetic DBC and did not occur in nondiabetic control CKC. The left-hand panel depicts a myelinated axon with ballooning superiorly (open arrow 1) and a ballooning inferiorly (open arrow 2) and note the interruption of the myelin sheath in mid axon (red dashed oval) and the cropped image insert from this region exploded in Microsoft paint with intact scale bar of $2 \mu \mathrm{m}$. Mid panel has open arrow pointing to balloon 1 and right-hand panel has open arrow pointing to balloon 2. Note the aberrant mitochondria pseudo-colored yellow with red outline. Magnification $\times 1500$; scale bar $=2 \mu \mathrm{m}$ far left, Magnification $\times 4000$; scale bar $=0.5 \mu \mathrm{m}$ in mid and far right-hand panels.

An unexpected finding within the cortical grey matter consisted of fragmented microtubules-neurofilaments and electron dense proteinaceous aggregations in three different unmyelinated axons at higher magnifications (Figure 5).
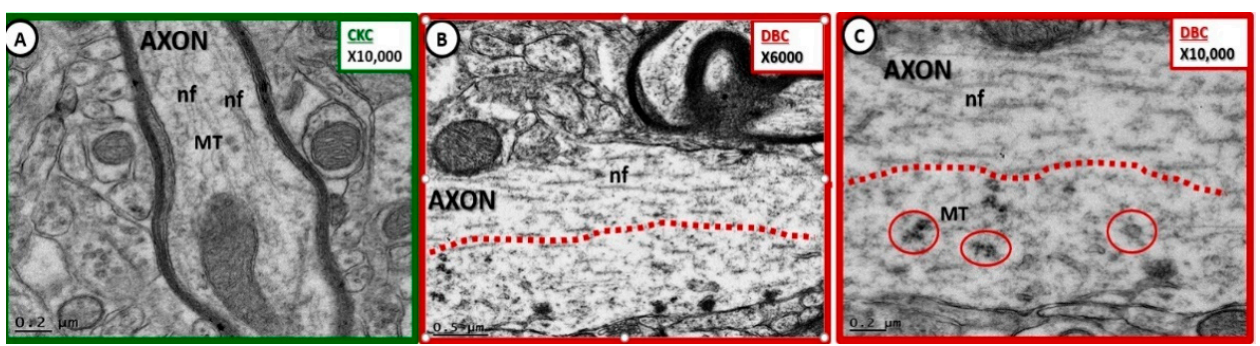

Figure 5. Larger axons, loss of microtubule neurofilament linearity, fragmentation and proteinaceous 
aggregation and deposition within axoplasm in some of diabetic DBC models. (B,C) Depict a loss of linearity of neurofilaments (nf) with fragmentation and aggregated proteinaceous electron dense deposits (within the lower half, below the red dashed line) axoplasm of diabetic DBC as compared to nondiabetic CKC controls (A). Some of the larger electron densities are microtubules (MT), while others could be protein aggregations. $(B, C)$ Are from different regions of the same unmyelinated axon. Note that the axon width of the DBC is $\sim 1.5 \mu \mathrm{m}$ as compared to $0.5 \mu \mathrm{m}$ in CKC. Magnifications $\times 10,000$ (A,C); scale bar $=0.2 \mu \mathrm{m}$. (B) Magnifications $\times 6000$; scale bar $=0.5 \mu \mathrm{m}$.

\subsection{Dysmyelination in the Transitional Zone between Cortical Layer VI and White Matter}

Due to the observations of splitting and separation of myelin lamellar units in the cortical grey matter, we decided to make deeper cuts to see if the transitional zone (TZ) between cortical layer and subcortical white matter also demonstrated abnormalities in myelinated axons since we were unable to examine any specific white matter tracts. One of the first things we noted was a decrease in neurovascular units in these regions as compared to cortical grey matter. These findings were anticipated, since it is known that the white matter has decreased capillary density as compared to cortical grey matter regions [8].

We noted a marked difference in myelinated axons in the TZ in the DBC models. These regions were observed to have a marked disarray of myelin with splitting-separation and collapse of axons from the myelin lamellar sheaths (Figure 6).

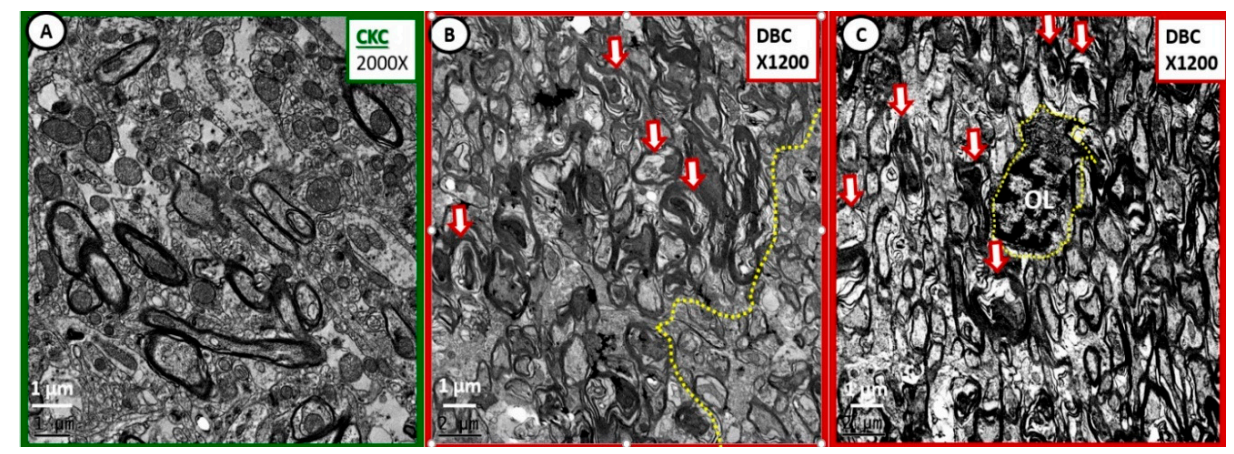

Figure 6. Extensive myelin remodeling disarray in diabetic DBC at deeper transition regions of the subcortical white matter regions. (A) Illustrates the normal appearance of the transitional subcortical white matter regions just deep to layer VI grey matter. (B,C) Depict markedly abnormal myelin remodeling in the diabetic DBC models. (B) Even demonstrates a demarcation between more severe remodeling to the left side of the demarcation (yellow dashed line) with more severe myelin splitting and axonal-axoplasm collapse (open red arrows) within the abnormal myelin lamellar sheaths. (C) Depicts a centrally located oligodendrocyte (OL outlined by yellow dashed line) that appears to be in the process of wrapping multiple axons with myelin. Importantly, note the very electron dense chromatin clumping - condensation within the nuclei and the very small amount of cytoplasm. (B,C) Depict marked disorganization of myelin lamella-dysmyelination with disordered myelin lamella, which is associated with splitting and separation of the myelin sheath lamella. Also, this region demonstrated marked abnormal remodeling changes of myelinated neurons in the diabetic DBC models as compared to control CKC models. Magnification $\times 2000$; scale bar $=1 \mu \mathrm{m}$ (A). Magnification $\times 1200$; scale bar $=1 \mu \mathrm{m}(\mathbf{B}, \mathbf{C})$.

Also, there were abnormalities of OLs in the diabetic DBC models in the transitional zone subcortical white matter between layers VI and deeper white matter (Figures 7 and 8). 

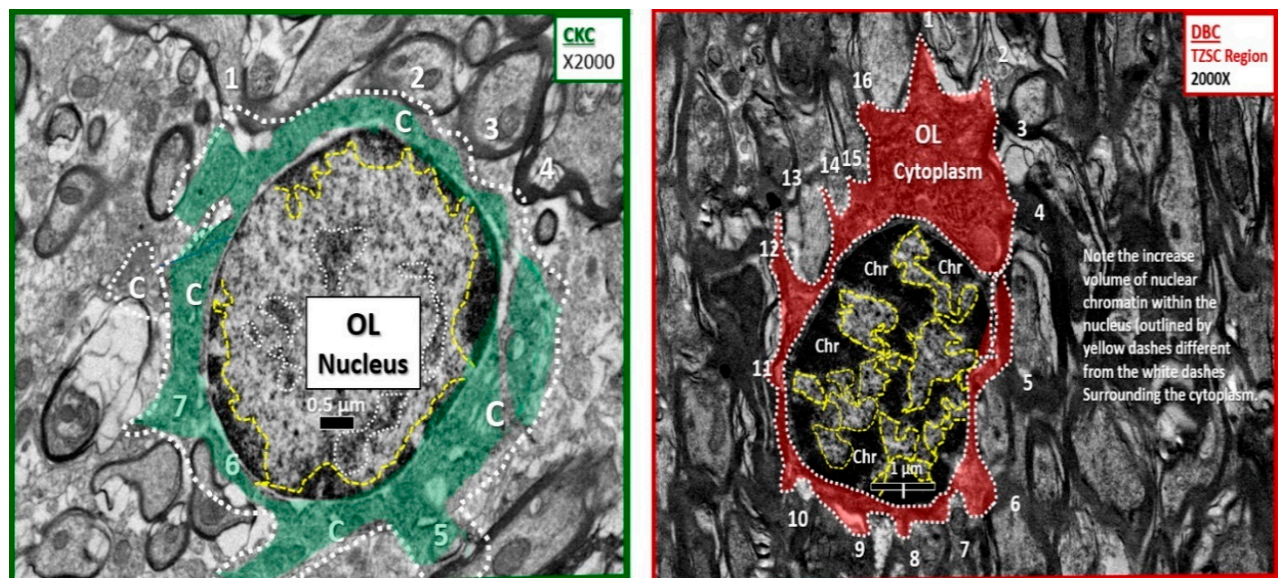

Figure 7. Increased chromatin condensation volume in nuclei of diabetic DBC models in the transitional zone subcortical regions as compared to nondiabetic CKC models. The nondiabetic CKC model to the left (cytoplasm pseudo-colored green and outlined with white dashed line) depicts an OL in the transitional zone subcortical (TZSC) region with surrounding myelinated axons and displays at least 1-7 numbered regions of myelination and maintenance zones. Note that the cytoplasm (C) in control models to the left has approximately seven regions of myelination as compared to diabetic DBC model to the right, which depicts up to 16 regions of myelination and maintenance zones with adjacent numerous electron dense myelinated axons (cytoplasm is pseudo-colored red with white dashed outline). Of great interest in the TZSC regions of the DBC, note that nuclear chromatin (Chr) is markedly increased (outlined by yellow dashed lines) in volume compared to nuclear volume when compared to the CKC nuclear chromatin on the left (outlined by yellow dashed lines). This was a consistent remodeling abnormality in the TZSC regions in the DBC models. Magnification $\times 2000$; bar $=0.5 \mu \mathrm{m}$ in $\mathrm{CKC}$ and $1 \mu \mathrm{m}$ in $\mathrm{DBC}$.
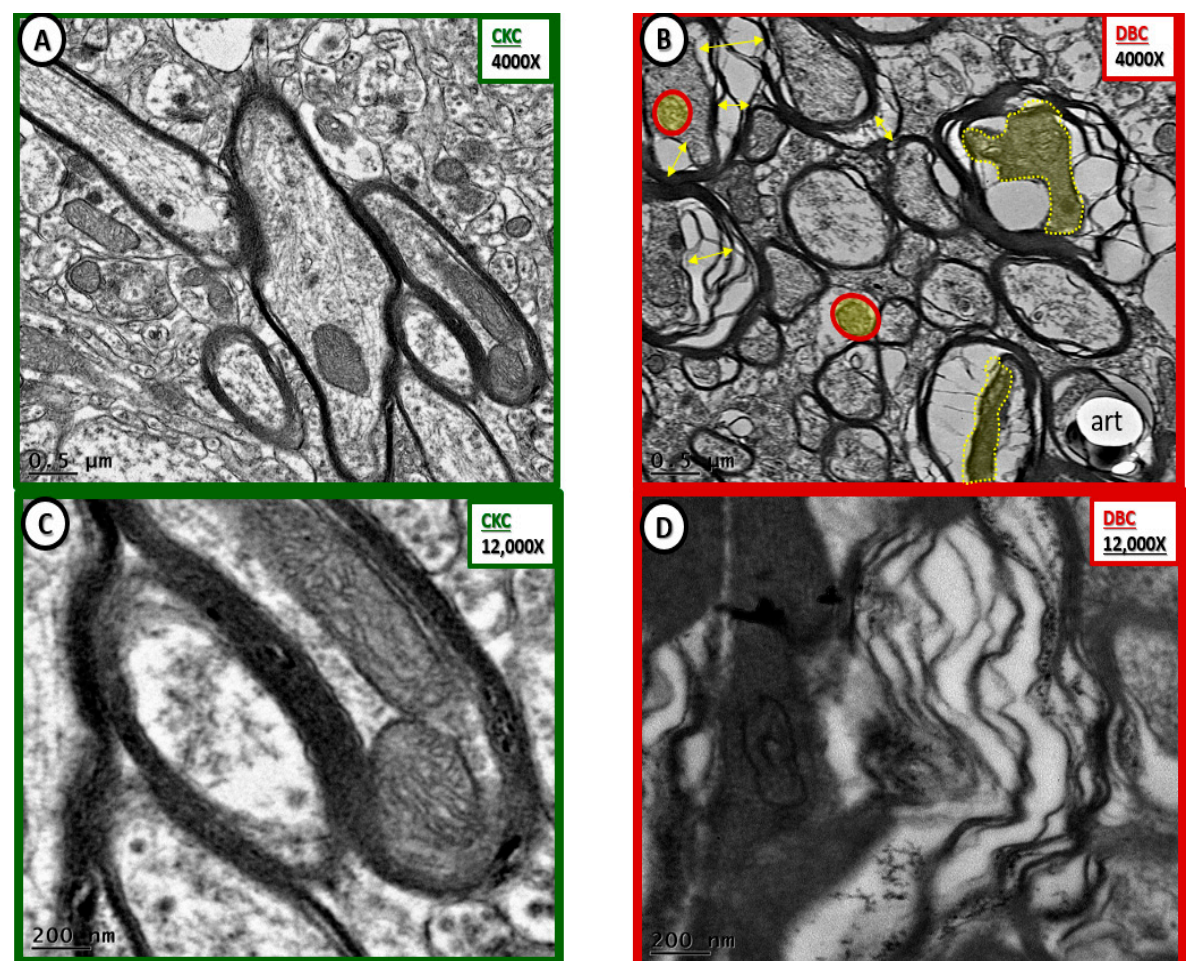

Figure 8. Markedly abnormal subcortical white matter myelin in diabetic DBC models. (C,D) Depict marked disorganization-disarrangement of the transitional zone subcortical white matter (SCWM) 
myelin and the neuronal axon in the diabetic DBC models. (B) Illustrates axon-axoplasm collapse (double arrows) in myelinated neurons. Also note that the neuronal axons become very electron dense once they collapse (pseudo-colored yellow and outlined by yellow dashed lines). Additionally, note the aberrant mitochondria (pseudo-colored yellow and outlined by red lines) as compared to the electron dense Mt with crista in the control CKC models (A,C). (D) Illustrates the abnormal myelin lamella with marked splitting and separation of myelin lamella. Magnification $\times 4000$; bar $=0.5 \mu \mathrm{m}$ in $(\mathbf{A}, \mathbf{B})$. Magnification $\times 12,000$; bar $=200 \mathrm{~nm}$ in $(\mathbf{C}, \mathbf{D})$. art: artifact.

Additionally noted was the collapse of the axons with myelin splitting and separation in the diabetic DBC models (Figures 9 and 10).
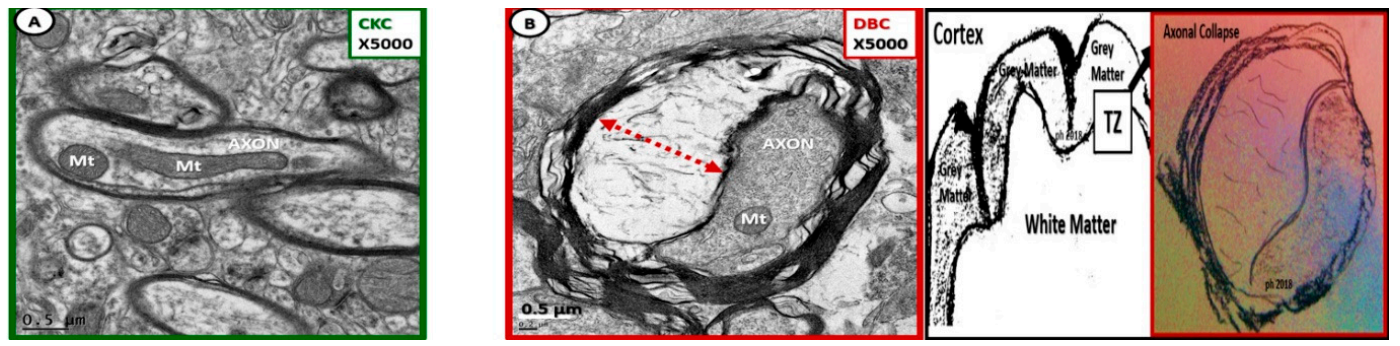

Figure 9. Axonal collapse in dysmyelinated neurons with splitting and separation transitional zones in diabetic DBC. Note in (B) that the collapse of the axon within the dysmyelinated neuron with splitting and separation of myelin lamella sheath in the transitional zones (TZ) of the subcortical white matter of the diabetic DBC as compared to the TZ non-collapsed axons in CKC models (A). Axonal collapse was markedly increased in the transitional zone regions as compared to the grey matter and not found in any of the CKC models. Note the region of the TZ in artistic rendering to far right of above figure. Magnification $\times 5000$; scale bar $=0.5 \mu \mathrm{m}$.
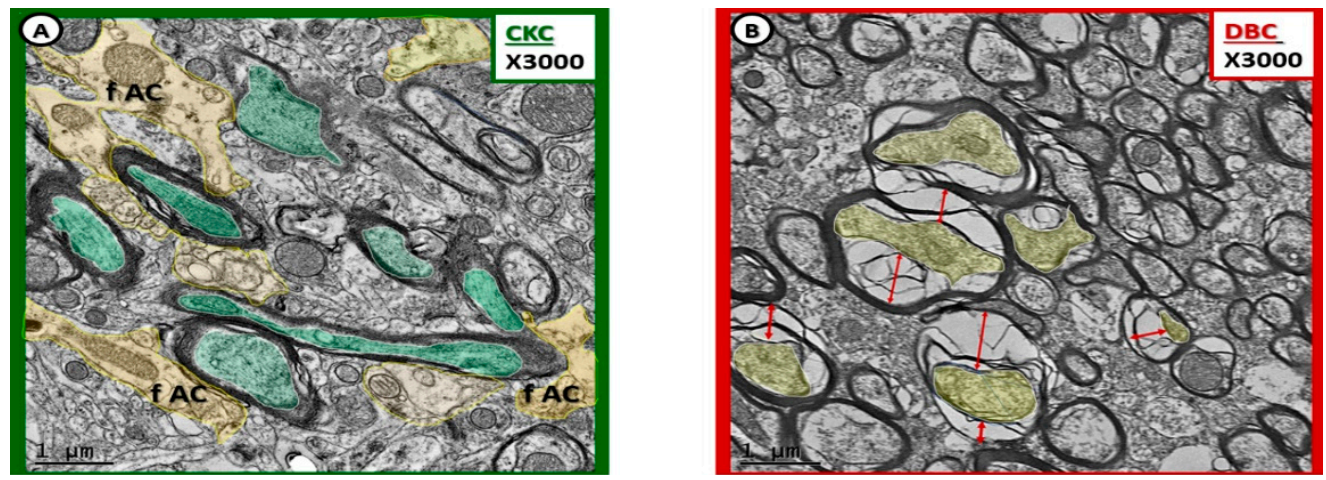

Figure 10. Myelin splitting, separation, and ballooning with axonal collapse in transitional zone in diabetic DBC models. (A) Depicts myelinated neurons with pseudo-colored green axons that fully occupy the myelin core. Also note the pseudo-colored yellow fibrous astrocyte (f AC) that are adjacent to the myelinated axons. (B) Demonstrates the splitting, separation, and ballooning of the myelin sheath resulting in axonal collapse in the diabetic DBC models in the transitional zone between layers $\mathrm{VI}$ and the subcortical white matter. Magnification $\times 3000$; scale bar $=1 \mu \mathrm{m}$ in $(\mathbf{A}, \mathbf{B})$.

\section{Discussion}

White matter is usually thought to be composed primarily by myelinated axons at the gross macroscopic level and is thought to be responsible for imparting a demarcation from the outer grey matter, which contains primarily neuronal cell bodies, glia, synaptic dendrites, and axon terminals of neurons. However, the white matter also contains numerous and varying percentages of unmyelinated 
axons: for example, the human corpus callosum may have up to $30 \%$ unmyelinated axons in contrast to the optic nerve where nearly all axons are myelinated [9].

Myelin wrapping and the final end-product of highly electron dense and compacted myelination of axonal ensheathment is quite complicated and illustrated in a simplified illustration (Figure 11). One can also view a more detailed 3D reconstruction with electron microscopy in the following reference for greater detail [10].

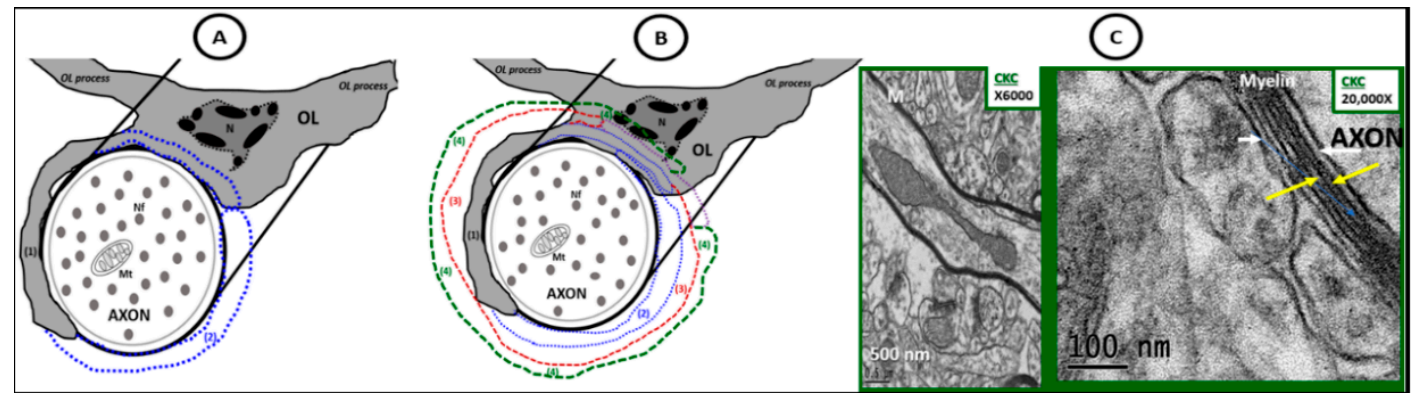

Figure 11. Possible mechanisms for oligodendrocyte myelination. (A) Depicts an illustration of the initial engagement of an OL and an unmyelinated axon with a protrusion of an oligodendrocyte cytoplasmic process (grey) beginning to extend and enwrap (blue dashed lines) of an unmyelinated neuron. (B) Depicts the possible multi-step process: (1) solid grey oligodendrocyte cytoplasmic process protrusion and engagement of the OL to the unmyelinated axon; (2) extension of the original oligodendrocyte cytoplasmic protrusion-blue dashed line; (3) another wrapping — red dashed line; (4) the most recent extension of wrapping - green dashed line. These previous steps may represent a common OL cellular mechanism of myelination. The multiple wrappings, which result in multilamellar myelin ensheathments are then bound tightly and compacted via the myelin basic protein plus other compacting proteins synthesized and secreted by oligodendrocytes that eventually develop into the highly electron dense tightly wrapped compacted myelinated neurons that we observe in transmission electron microscopic images in control CKC models as depicted in the control CKC model in (C). This mechanism does not explain for the lateral extension of the internodal myelin. Note that in (C) there is present some separation of myelinated lamella as a result of high magnification $\times 20,000$; scale bar $=100 \mathrm{~nm}$ that is not recognized at lower magnification. This higher magnification delineates the electron dense (dense lines-yellow arrows) and the electron lucent (intraperiod lines-white arrows). M: mitochondria; N: nucleus of oligodendrocyte; Nf: neurofilament; OL: oligodendrocyte.

The images in the results section may provide additional concepts and improve our understanding of the oligodendrocyte and myelin formation in both the grey matter and transitional zone between the grey matter and the white matter (Figure 9 far right-artistic rendition) in the DBC models. Indeed, it is obvious that similar to other glial cells, oligodendrocytes respond with cellular and tissue remodeling to the multiple toxicities associated with obesity, insulin resistance, and glucotoxicity when the receptor for leptin is deficient as in the DBC model. Of note, OLs not only effect myelination and their maintenance to individual axons, but also may be important to entire neuronal networks as a result of shape-shifting-remodeling changes of their ultrastructural morphology including myelin splitting, ballooning, and axonal collapse. As a result of leptin receptor deficiency, OLs may remodel their ultrastructure to result in changes of CNS behavioral effects of depression and cognitive impairment. Indeed, OLs are dynamic, adaptive, and plastic and are certainly capable of responding via a response to injury mechanism and even aid in the development of abnormal brain behavior in this diabetic DBC model [11].

The ultrastructural remodeling in the DBC models (Figure 5) could represent an early axoplasm remodeling change of fragmentation of neurofibrillary tangles that are known to be associated with increased possibility of tau pathology in the $\mathrm{db} / \mathrm{db}$ models of leptin resistance and type 2 diabetes [11]. 
Additionally, others have shown similar remodeling changes with fragmentation of axonal neurofilaments in both myelinated and unmyelinated axons similar to what we observed (only in unmyelinated neuronal axons) in streptozotocin induced type 1 diabetic rats [12]. However, the unmyelinated axon we demonstrated (Figure 5) may have depicted some proteinaceous aggregation in DBC models in addition to microtubules.

In Figures 2-4 (cortical grey matter) and Figures 6-10 (subcortical white matter transitional zone) we demonstrated splitting, separation, and ballooning of myelinated neurons. These changes have been previously described by others in models of aging $[13,14]$. Peters et al. state that there are four basic changes in myelin in aged monkey brains, which consist of local splitting, ballooning, redundant myelin, and double myelin sheaths-duplication, which we have depicted in our DBC as compared to the CKC models [13]. These findings seem to also corroborate Parts I and II of this series in that the oligodendrocyte and myelin remodeling changes suggests an accelerated and premature aging process in the diabetic DBC as compared to the control CKC models [14].

In regard to the transitional zone between cortical grey and white matter, the subcortical white matter may represent a region in the brain that is less vascularized [15-17]. Interestingly, our observational findings regarding vascularity in the cortex revealed that there are usually 2-4 neurovascular units (NVU) capillaries per grid examined and in the transitional zone described there was usually only one NVU capillary at the most with a preponderance of no NVU capillaries per grid examined. Additionally, an older paper states the following, "it is possible that the junction between cortex and white matter is relatively avascular, and in this respect this area may be similar to the particular periventricular regions" [18].

Oligodendrocytes and myelination are not static and readily adapt to their environment. The previously described alterations in myelin remodeling in the diabetic DBC models may be detrimental and could impair cognition by the slowing of conduction rates of information-action potential in white matter tracts to distant regions, which could result in a loss of synchronicity not only in individual neurons, but to entire neuronal networks.

\subsection{Vascular Stiffness}

Vascular stiffness including thoracic aorta or carotid artery not only affects the capillaries of the classical end-organs of diabetes (retinopathy, neuropathy, nephropathy, and cardiomyopathy) due to abnormal pulsatile mechanical forces that are associated with microvascular damage and remodeling but may also affect the brain in diabetic cognopathy. In particular, this same cohort that we studied $(\mathrm{db} / \mathrm{db}-\mathrm{DBC})$ in these three-part series (Parts I, II, and III) has been previously demonstrated to have aortic vascular stiffening [19]. The capillary neurovascular remodeling changes described in Part I could affect the possible diminished microvessels in the transition zones as well as the ones we demonstrated within the grey matter and could decrease the cerebral blood flow to the transitional zone regions as well as the subcortical white matter [20-22]. These NVU remodeling changes could be an additional risk to the development of dysmyelination as we have observed in both the grey matter and transitional zones as described in this paper. The decreased CBF in the TZ and the subcortical white matter (SCWM) could be even more devastating due the possible decreased capillary density found in these regions in comparison to the grey matter [18] (Figure 12). 


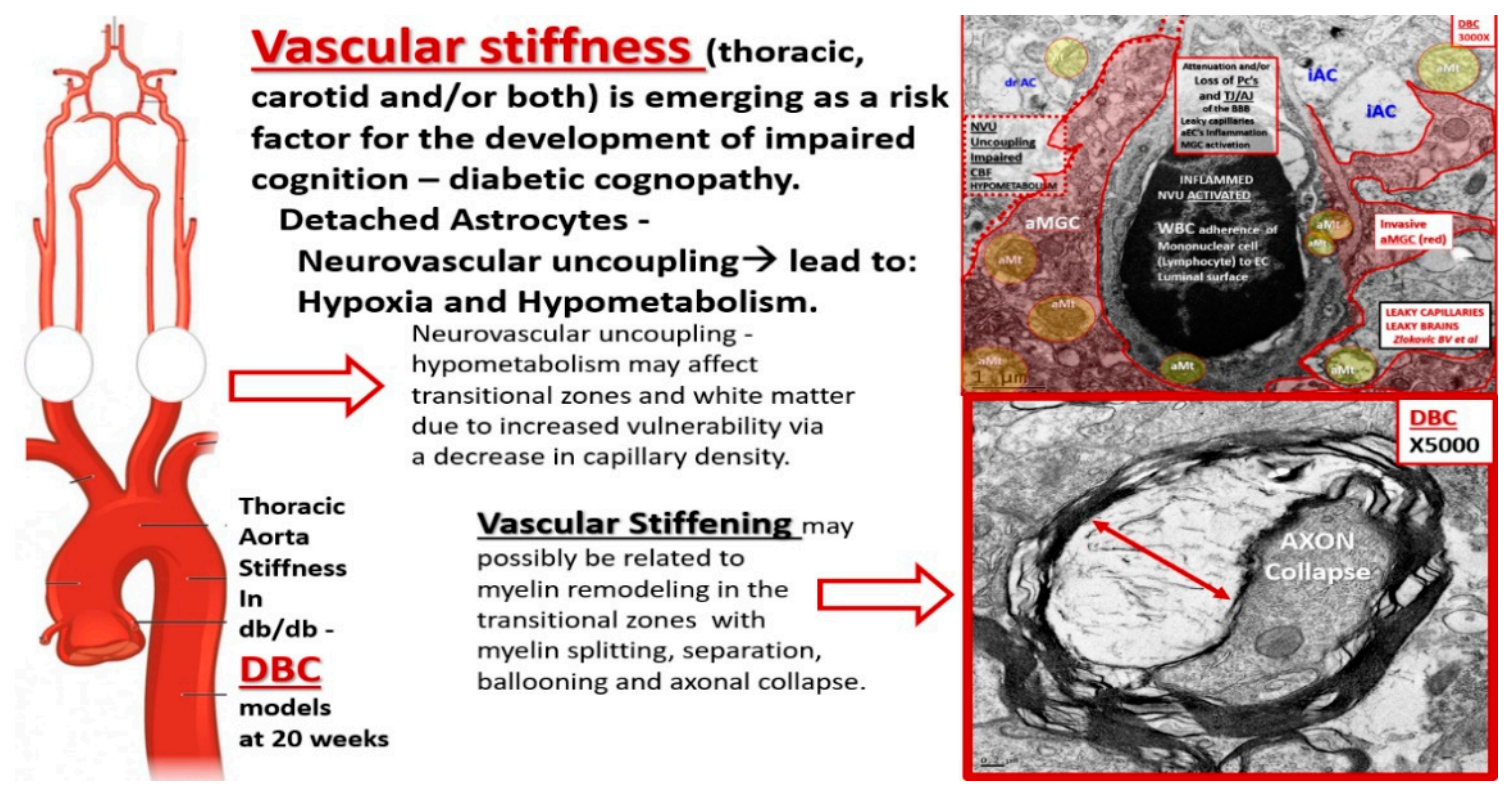

Figure 12. Montage of images demonstrating that vascular stiffness in the thoracic or carotid arteries may result in capillary neurovascular unit remodeling and the possible relationship to the development of dysmyelination in the diabetic DBC models. On the far left are depictions of the thoracic aorta, carotids, and brain circulatory system that are known to experience vascular stiffness in the diabetic DBC model [19]. The open arrow then points to the fact that there may be neurovascular uncoupling, which decreases the cerebral blood flow and could result in hypometabolism not only in the cortical grey matter but also in the vulnerable transitional zone. Cerebral flow pulsatility is augmented in type 2 diabetes mellitus (T2DM) [17] and this has been associated with markers of cerebral damage, such as white matter hyperintensities in T2DM. Thus, thoracic or carotid artery stiffness could result in dysmyelination and in turn the dysmyelination could slow the delivery of information-action potential without proper synchronicity and result in impaired cognition.

Importantly, diabetes is not only a risk factor for stroke, but also a risk factor for the development of white matter lesions [23]. Yotomi Y. et al. have demonstrated that the $\mathrm{db} / \mathrm{db}$ diabetic model had greater severity of white matter damage that was associated with decreased proliferation and survival of oligodendrocyte progenitor cell [23].

\subsection{Axon Initial Segment Shortening in db/db Diabetic DBC Models}

Keiichiro Susuki's group has recently demonstrated that diabetic $\mathrm{db} / \mathrm{db}$ brains ( $>10$ weeks old) are known to abnormally remodel their neuronal axons, which result in axon initial segment (AIS) shortening [24]. While we were unable to demonstrate shortening of the axon initial segment via our ultrastructural observations, we wish to demonstrate the adjacent nanometer proximity of the astrocyte, microglia to the pyramidal neurons. The activation and detachment of the astrocyte and/or activation of microglia provides the potential for cellular crosstalk via ultrastructural astrocyte and microglia, which may be a contributing factor for remodeling change in DBC models (Figure 13). Because of the adjacent nanometer proximity between the glia (astrocytes and microglia) and neurons, we hypothesize that when microglia are activated as in our DBC model their aberrant leaky mitochondria could result in increased oxidative/nitrosative stress such that the AIS could be shortened secondary to excess reactive oxygen/reactive nitrogen species (ROS/RNS) derived from activated microglia, intracellular calcium accumulation, and AIS shortening [24]. Additionally, we observed abnormalities in myelin remodeling-dysmyelination, which could act in synergy with AIS shortening and could contribute to an increased loss of synchronicity of action potential to distant sites and result in dysfunctional 
signaling to receiving neurons and neuronal networks. These changes could increase the possibility of impaired cognition as noted in the diabetic $\mathrm{db} / \mathrm{db}$ DBC models [24-27].

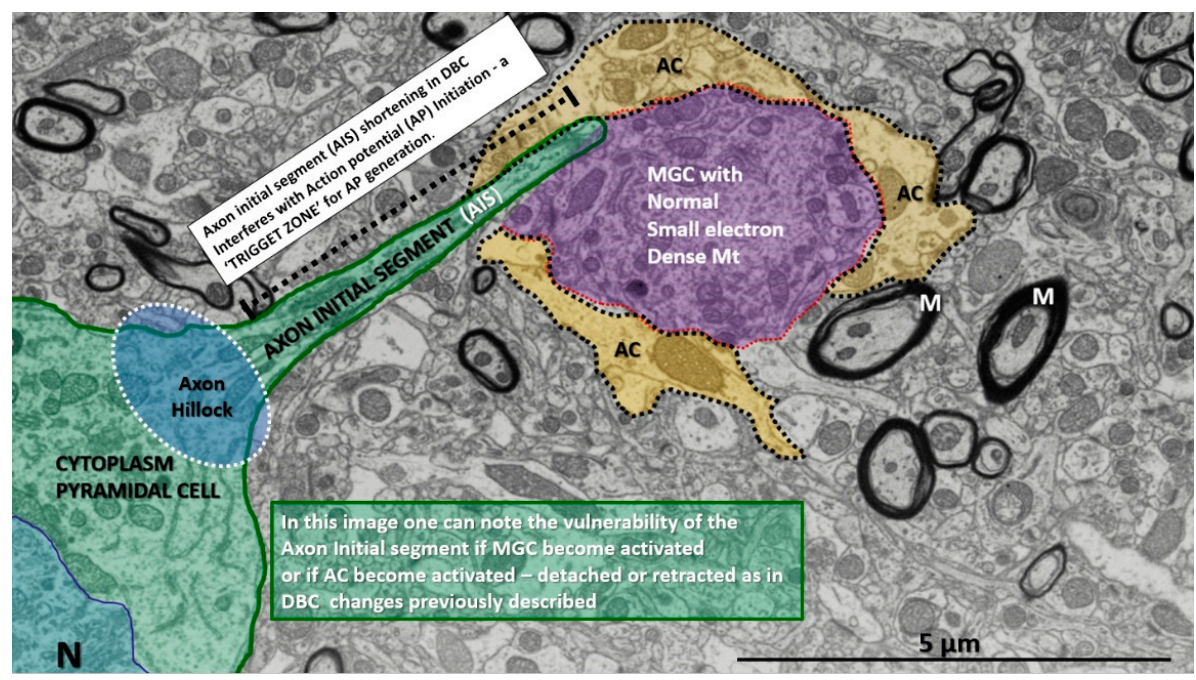

Figure 13. Pyramidal cell demonstrating the normal axon initial segment (AIS) in a control model. Note the cytoplasm of this pyramidal cell has an axon hillock region and also the axon initial segment region as the axon protrudes from the soma of the pyramidal cell. M: myelin; MGC: microglial cell; $\mathrm{N}$ : pyramidal nucleus; $\mathrm{AP}=$ action potential. Scale bar $=5 \mu \mathrm{m}$.

Interestingly, we observed ballooning of some axons with evidence of the neuroprotective astrocyte being detached from the axon and retracted, similar to the neurovascular unit astrocytes in Part I of this series (Figure 14) [5]. Astrocytes are important in protecting the pyramidal neuronal axons in layer III and throughout the cortical grey matter, transitional zones and subcortical white matter [28].

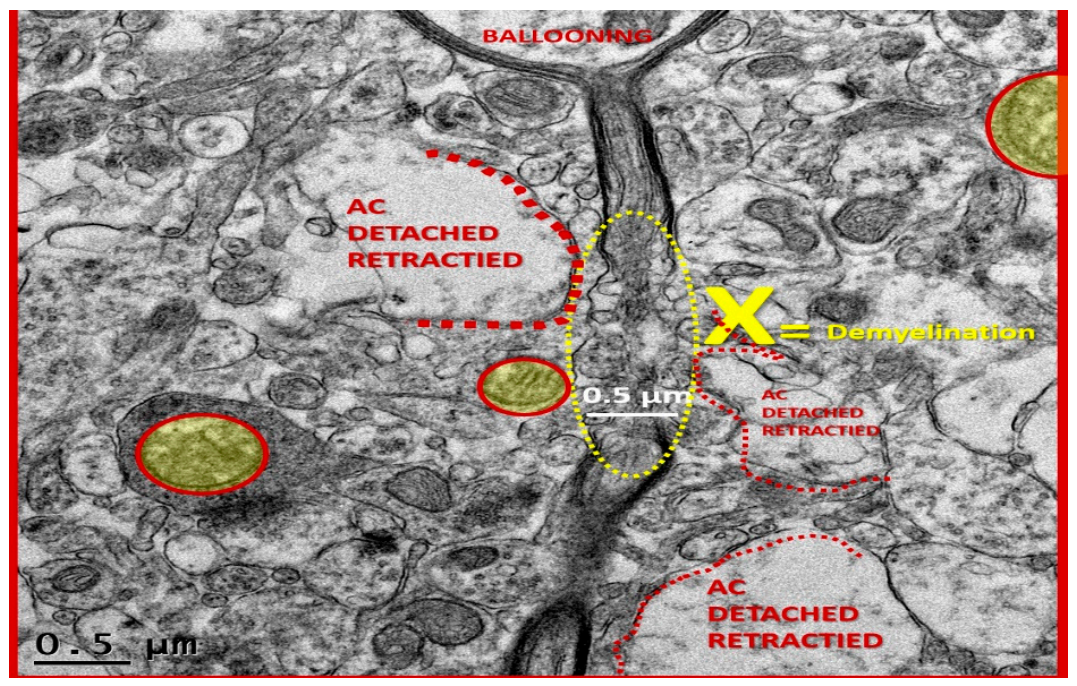

Figure 14. Detached and retracted astrocytes from a ballooning pyramidal neuronal axon in layer III of cortical grey matter. This is a higher magnification of previous image Figure $4 \times 1500$. In this image one can observe that there is a region marked with the letter $\mathrm{X}$ (yellow) where there is an area of myelin loss (demyelination) in a ballooned axon within layer III of the cortical grey matter. Importantly, note the detachment and retraction of astrocytes (AC) from the neuronal axon (defined in red lettering and by dashed lines). Also note the presence of at least three aberrant mitochondria in this region. Magnification $\times 4000$; scale bar $=0.5 \mu \mathrm{m}$. 
In summary, we have attempted to place this three-part series (Parts I, II, and III) regarding neurovascular unit and glia (astrocyte, microglia, and oligodendrocyte) remodeling changes in perspective to a more clinical setting as they pertain to type 2 diabetes mellitus in humans (Figure 15).

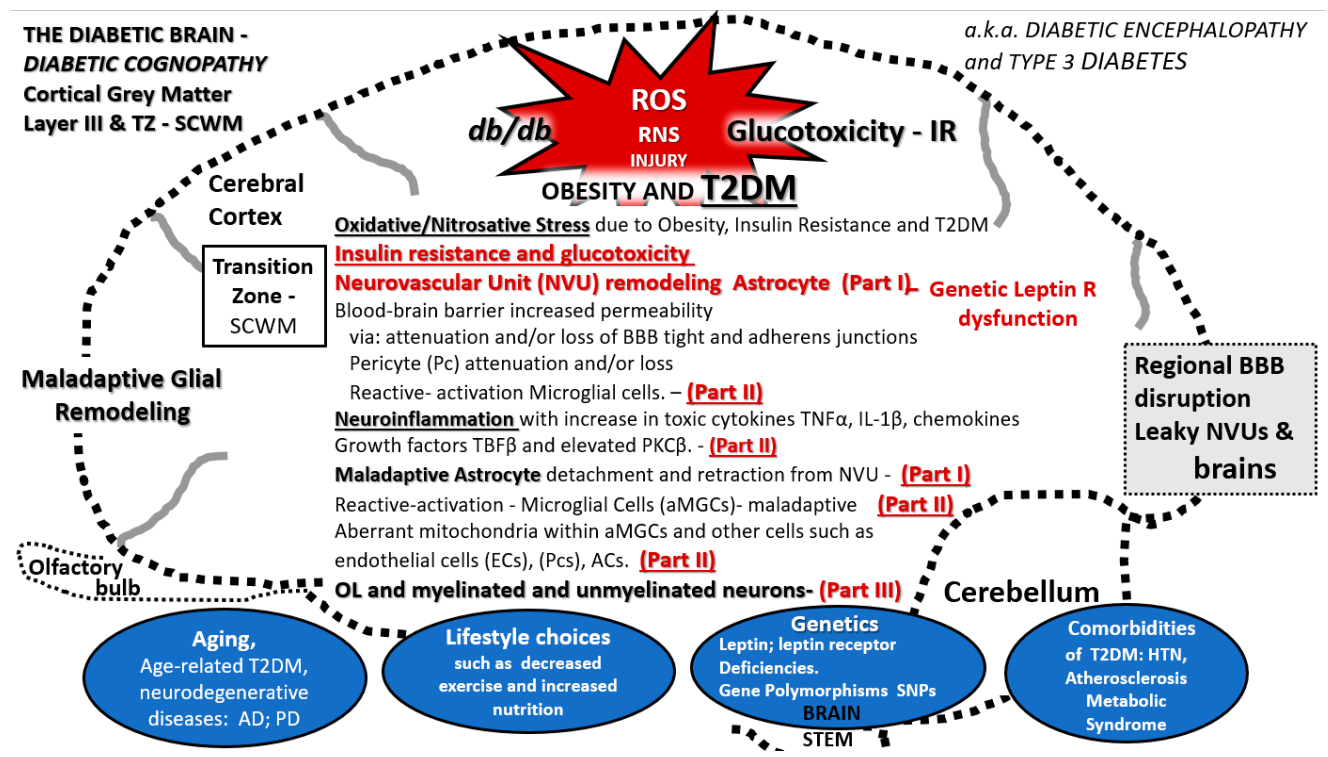

Figure 15. Multiple predisposing risk factors and metabolic and structural defects in DBC models. This figure depicts the multiple predisposing risk factors, metabolic and our ultrastructural remodeling that we have shared regarding the neurovascular unit and glia including the astrocyte (AC), microglia, and oligodendrocyte in this three-part series. The blue ovals at the bottom of the image depict the multiple situations that might give rise to the remodeling changes and how they are related to each of the three-part series as Part I, Part II, and Part III publications as follows: Aging, lifestyle, environment, genetics-particularly the leptin receptor deficiencies in the DBC and also the numerous single nucleotide polymorphisms (SNPs) in humans, and comorbidities associated with obesity (metabolic syndrome and accelerated atherosclerosis). a.k.a: also known as; AD: Alzheimer's disease; aMGC: activated microglia cell; BBB: blood-brain barrier; db/db: DBC; HTN: hypertension; IL-1 $\beta$ : interleukin 1 beta; IR: insulin resistance; NVU: neurovascular unit; OL: oligodendrocyte; PD: Parkinson's disease; PKC $\beta$ : protein kinase C beta; R: receptor; ROS/RNS: reactive oxygen/reactive nitrogen species; SCWM: subcortical white matter; T2DM: type 2 diabetes mellitus; TGF $\beta$ : transforming growth factor beta; $\mathrm{TNF} \alpha$ : tumor necrosis factor alpha; TZ: transition zone.

\section{Future Directions}

Although we have not yet been able to study oligodendrocyte and myelin extensively with focused ion beam/scanning electron microscopy technology as we have previously done with the microglia in supplement videos in Part II of this series [29], we look forward to this possibility in due time such that we may be able to better understand their 3D stacks to create oligodendrocyte and myelin videos. Additionally, we hope to study oligodendrocytes and myelin remodeling specifically in the corpus callosum and/or optic nerves and how they relate to elongated white matter tracts. Indeed, these are exciting times to study the ultrastructural remodeling changes in the glia.

Author Contributions: M.R.H. and V.G.D. conceptualized the study; M.R.H. performed the image collection and interpretation; D.G.G. prepared the tissue specimens for transmission electron microscopic studies and assisted M.R.H.; M.R.H. prepared the manuscript; A.A. collected tissue specimens; V.G.D., A.A., and D.G.G. assisted in editing.

Funding: This study was supported by an internal grant entitled Excellence in Electron Microscopy grant and issued by the University of Missouri Electron Microscopy Core and Office of Research. No external funding was available. 
Acknowledgments: Authors wish to thank Tommi White of the University of Missouri Electron Microscopy Core Facility. Authors also wish to thank our mentor James R. Sowers, who inspired us to interrogate, characterize, and understand the secrets that the tissues behold.

Conflicts of Interest: The authors declare no conflict of interest.

\section{References}

1. Michalski, J.P.; Kothary, R. Oligodendrocytes in a nutshell. Front. Cell. Neurosci. 2015, 9, 340. [CrossRef] [PubMed]

2. Peters, A.; Palay, S.L.; Webster, H. The Fine Structure of the Nervous System-Neurons and Their Supportive Cells-The Cellular Sheaths of Neurons, 3rd ed.; Oxford University Press: New York, NY, USA, 1991; pp. $212-261$. ISBN 0-19-506571-9.

3. Pukos, N.; Yoseph, R.; McTigue, D.M. To Be or Not to Be: Environmental Factors that Drive Myelin Formation during Development and after CNS Trauma. Neuroglia 2018, 1, 63-90. [CrossRef]

4. Boullerne, A.I. The history of myelin. Exp. Neurol. 2016, 283, 431-445. [CrossRef] [PubMed]

5. Hayden, M.R.; Grant, D.; Aroor, A.; Demarco, V.G. Ultrastructural Remodeling of The Neurovascular Unit in The Female Diabetic db/db Model-Part I: Astrocyte. Neuroglia 2018, 1, 220-244. [CrossRef]

6. Montagne, A.; Nikolakopoulou, A.M.; Zhao, Z.; Sagare, A.P.; Si, G.; Lazic, D.; Barnes, S.R.; Daianu, M.; Ramanathan, A.; Go, A.; et al. Pericyte degeneration causes white matter dysfunction in the mouse central nervous system. Nat. Med. 2018, 24, 326-337. [CrossRef] [PubMed]

7. Reijmer, Y.D.; Brundel, M.; de Bresser, J.; Kappelle, L.J.; Leemans, A.; Biessels, G.J. Utrecht Vascular Cognitive Impairment Study Group. Microstructural white matter abnormalities and cognitive functioning in type 2 diabetes: A diffusion tensor imaging study. Diabetes Care 2013, 36, 137-144. [CrossRef] [PubMed]

8. Cavaglia, M.; Dombrowski, S.M.; Drazba, J.; Vasanji, A.; Bokesch, P.M.; Janigro, D. Regional variation in brain capillary density and vascular response to ischemia. Br. Res. 2001, 910, 81-93. [CrossRef]

9. Walhovd, K.B.; Johansen-Berg, H.; Káradóttir, R.T. Unraveling the secrets of white matter-Bridging the gap between cellular, animal and human imaging studies. Neuroscience 2014, 276, 2-13. [CrossRef] [PubMed]

10. Snaidero, N.; Möbius, W.; Czopka, T.; Hekking, L.H.; Mathisen, C.; Verkleij, D.; Goebbels, S.; Edgar, J.; Merkler, D.; Lyons, D.A.; et al. Myelin membrane wrapping of CNS axons by PI(3,4,5)P3-dependent polarized growth at the inner tongue. Cell 2014, 156, 277-290. [CrossRef] [PubMed]

11. Platt, T.L.; Beckett, T.L.; Kohler, K.; Niedowicz, D.M.; Murphy, M.P. Obesity, diabetes, and leptin resistance promote tau pathology in a mouse model of disease. Neuroscience 2016, 315, 162-174. [CrossRef] [PubMed]

12. Hernández-Fonseca, J.P.; Rincón, J.; Pedreañez, A.; Viera, N.; Arcaya, J.L.; Carrizo, E.; Mosquera, J. Structural and ultrastructural analysis of cerebral cortex, cerebellum, and hypothalamus from diabetic rats. Exp. Diabetes Res. 2009, 2009, 329632. [CrossRef] [PubMed]

13. Peters, A.; Moss, M.B.; Sethares, C. Effects of aging on myelinated nerve fibers in monkey primary visual cortex. J. Comp. Neurol. 2000, 419, 364-376. [CrossRef]

14. Wrighten, S.A.; Piroli, G.G.; Grillo, C.A.; Reagan, L.P. A look inside the diabetic brain: Contributors to diabetes-induced brain aging. Biochim. Biophys. Acta 2009, 1792, 444-453. [CrossRef] [PubMed]

15. Bowley, M.P.; Cabral, H.; Rosene, D.L.; Peters, A. Age changes in myelinated nerve fibers of the cingulate bundle and corpus callosum in the rhesus monkey. J. Comp. Neurol. 2010, 518, 3046-3064. [CrossRef] [PubMed]

16. Sosa, S.M.; Smith, K.J. Understanding a role for hypoxia in lesion formation and location in the deep and periventricular white matter in small vessel disease and multiple sclerosis. Clin. Sci. 2017, 131, 2503-2524. [CrossRef] [PubMed]

17. Nonaka, H.; Akima, M.; Hatori, T.; Nagayama, T.; Zhang, Z.; Ihara, F. Microvasculature of the human cerebral white matter: Arteries of the deep white matter. Neuropathology 2003, 23, 111-118. [CrossRef] [PubMed]

18. Brownell, B.; Hughes, J.T. The distribution of plaques in the cerebrum in multiple sclerosis. J. Neurol. Neurosurg. Psychiatr. 1962, 25, 315-320. [CrossRef]

19. Aroor, A.R.; Das, N.A.; Carpenter, A.J.; Habibi, J.; Jia, G.; Ramirez-Perez, F.I.; Martinez-Lemus, L.; Manrique-Acevedo, C.M.; Hayden, M.R.; Duta, C.; et al. Glycemic control by the SGLT2 inhibitor empagliflozin decreases aortic stiffness, renal resistivity index and kidney injury. Cardiovasc. Diabet. 2018, 17, 108. [CrossRef] [PubMed] 
20. Cooper, L.L.; Mitchell, G.F. Aortic Stiffness, Cerebrovascular Dysfunction, and Memory. Pulse 2016, 4, 69-77. [CrossRef] [PubMed]

21. Heffernan, K.S. Carotid artery stiffness and cognitive function in adults with and without type 2 diabetes: Extracranial contribution to an intracranial problem? Atherosclerosis 2016, 253, 268-269. [CrossRef] [PubMed]

22. Lee, K.O.; Lee, K.Y.; Lee, S.Y.; Ahn, C.W.; Park, J.S. Lacunar infarction in type 2 diabetes is associated with an elevated intracranial arterial pulsatility index. Yonsei Med. J. 2007, 48, 802-806. [CrossRef] [PubMed]

23. Yatomi, Y.; Tanaka, R.; Shimada, Y.; Yamashiro, K.; Liu, M.; Mitome-Mishima, Y.; Miyamoto, N.; Ueno, Y.; Urabe, T.; Hattori, N. Type 2 diabetes reduces the proliferation and survival of oligodendrocyte progenitor cells in ischemia white matter lesions. Neuroscience 2015, 289, 214-223. [CrossRef] [PubMed]

24. Yermakov, L.M.; Drouet, D.E.; Griggs, R.B.; Khalid, M.; Elased, K.M.; Susuki, K. Type 2 Diabetes Leads to Axon Initial Segment Shortening in db/db Mice. Front. Cell. Neurosci. 2018, 12, 146. [CrossRef] [PubMed]

25. Benusa, S.D.; George, N.M.; Sword, B.A.; DeVries, G.H.; Dupree, J.L. Acute neuroinflammation induces AIS structural plasticity in a NOX2-dependent manner. J. Neuroinflamm. 2017, 14, 116. [CrossRef] [PubMed]

26. Rasband, M.N. The axon initial segment and the maintenance of neuronal polarity. Nat. Rev. Neurosci. 2010, 11, 552-562. [CrossRef] [PubMed]

27. Bender, K.J.; Trussell, L.O. The physiology of the axon initial segment. Annu. Rev. Neurosci. 2012, 35, $249-265$. [CrossRef] [PubMed]

28. Bélanger, M.; Magistretti, P.J. The role of astroglia in neuroprotection. Dialog. Clin. Neurosci. 2009, 11, $281-295$.

29. Hayden, M.R.; Grant, D.; Aroor, A.; Demarco, V.G. Ultrastructural Remodeling of The Neurovascular Unit in The Female Diabetic db/db Model-Part II: Microglia and Mitochondria. Neuroglia 2018, 1, 311-326. [CrossRef]

(C) 2018 by the authors. Licensee MDPI, Basel, Switzerland. This article is an open access article distributed under the terms and conditions of the Creative Commons Attribution (CC BY) license (http:/ / creativecommons.org/licenses/by/4.0/). 